\title{
Diagnosis of Buruli ulcer disease in Nigeria using IS2404-Based Nested PCR
}

\author{
Vincent P. Gyang ${ }^{1 *}$, Timothy Nwafor ${ }^{1}$, Susan I. Alexander ${ }^{2}$, Adewale Oke ${ }^{3}$, \\ Olaoluwa P. Akinwale ${ }^{1}$ \\ ${ }^{I}$ Department of Public Health and Epidemiology, Nigerian Institute of Medical Research, \\ Lagos, Nigeria. \\ ${ }^{2}$ Department of Zoology, University of Lagos, Akoka, Lagos State, Nigeria \\ ${ }^{3}$ Department of Biological Sciences, College of Natural Sciences, Redeemers University, Ede, \\ Nigeria.
}

*Correspondence should be addressed to Vincent P. Gyang: gyangvince@yahoo.com

Received 8th February 2021; Revised 4th March 2021; Accepted 6th March 2021

(C) 2021 Gyang et al. Licensee Pan African Journal of Life Sciences an official publication of Faculty of Basic Medical Sciences, Ladoke Akintola University of Technology, Ogbomoso. This is an Open Access article distributed under the terms of the Creative commons Attribution License (https://creativecommons.org/licenses/BY/4.0), which permits unrestricted use, distribution, and reproduction in any medium, provided the original work is properly cited.

\begin{abstract}
Background: Buruli ulcer is a chronic, indolent, necrotizing infectious disease of the skin and soft tissues characterized by the formation of large ulcers, often in the arms or legs. Nigeria is a Buruli ulcer disease (BUD) endemic country with its control programme still in infancy. As a result, samples are sent to laboratories outside the country. Some patients go to neighbouring countries with more established programmes for polymerase chain reaction (PCR) as a basis of diagnosis and treatment. Hence, this study was embarked upon to assist the national control programme in overcoming the PCR diagnosis test challenge.

Method: This was a cross-sectional and community-based type of study of Buruli ulcer patients from 15 states, mostly from southern Nigeria; Abia, Akwa Ibom, Anambra, Bayelsa, Cross Rivers, Delta, Ebonyi, Ekiti, Enugu, Imo, Lagos, Ogun, Ondo, Osun, Rivers and the Federal Capital Territory (FCT) Abuja. Swab and Fine Needle Aspiration (FNA) samples were received from January 2016 to June 2018. DNA was extracted, and each sample was subjected to IS2404-based nested PCR.

Results: Out of 920 samples received, 427 (46.4\%) were IS2404-based nested PCR positive. Of which 204 were males, and 223 were females. The patients' mean age was $35.7 \pm 19.94$, with 172 (18.7\%) being children $\leq$ 15 years, while $748(81.3 \%)$ were $\geq 15$ years. During the study period, the highest number of samples, 171 $(18.6 \%)$ and $170(18.5 \%)$ were received from Cross Rivers and Delta states, respectively. In contrast, the least number of samples, $2(0.2 \%)$ and $3(0.3 \%)$, came from Ekiti and Lagos states, respectively.

Conclusion: This is the first in-country PCR confirmed diagnosis of a large cohort of BU patients. The results show a high prevalence in southern Nigeria, which is an indicator of high transmission. Our findings suggest the need for a prompt intervention by the government by providing the needed health facilities and education for the communities.
\end{abstract}

Keywords: Mycobacterium ulcerans, IS2402-Based Nested PCR, Confirmatory laboratory diagnosis 


\section{INTRODUCTION}

Mycobacterium ulcerans, the causative pathogen of Buruli ulcer disease (BUD), ranks third among the most common mycobacteria infection worldwide, after tuberculosis and leprosy [1]. Though in some affected communities in the west and central Africa, BUD incidence rates surpass the other two [2]. With early cases first described in Buruli county of Uganda [3], the World Health Organization (WHO) in 1998 declared Buruli ulcer an emerging skin disease of public concern. The disease has now been reported in over 33 countries in Africa, Asia, America, and West Pacific [1], with most cases currently in West Africa [4].

Buruli ulcer is a disfiguring and occasionally disabling cutaneous disease characterized by chronic necrosis of subcutaneous tissue, with bones affected in some cases. At the onset of infection, it appears as a painless insect bite or pimple which progresses slowly destroying subcutaneous tissues leading to an extensive skin peel off, then the characteristic ulcer with undermined edges [5]. The cytotoxic and immunosuppressive activities of mycolactone, a polyketide toxin produced by M. ulcerans is responsible for the necrosis of tissue which distinguishes it from other mycobacteria. This has been suggested as a possible diagnostic target but for its lipid nature, making it poorly immunogenic [6].

M. ulcerans is an environmental bacterium often found in swampy and humid areas with stagnant lakes or slowflowing streams in tropical and subtropical regions globally $[7,8]$. However, some cases have been reported without exposure to wetlands. The establishment of a specific mode of transmission to humans remains elusive, with many theories proposed. However, established risk factors include; living in BU endemic areas, previous trauma to the skin, and lack of wearing protective footwear $[9,10]$. M. ulcerans have been found in aquatic bugs and mosquito, fish, aquatic plants, and Acanthamoeba species, but no conclusion about the transmission mode has been drawn yet $[11,12,13]$.

The first time Nigeria notified WHO of BUD cases was in 2006, after a collaborative assessment by a team from Benin republic and $\mathrm{WHO}$, which investigated the BUD situation in the country with the aim of establishing endemic areas [14]. Historically, Gray et al in 1967 described the first cases of BUD in Nigeria in four patients residing in Benue state. Then in 1976 another 24 patients were described around Ibadan in Oyo state [15]. Since then, over two decades passed before investigations on BUD picked up again in the country between 1998 and 2000 when samples from the Leprosy and Tuberculosis Hospital in Moniaya-Ogoja, CrossRivers state, were sent to the Institute of Tropical Medicine in Belgium for PCR confirmation.

Diagnosis of BUD is currently done using four methods: PCR, usually IS2404 amplification, microscopy (ZN stain) for acid-fast bacilli detection, culture for the isolation of viable organisms, and histopathology. The IS2404 PCR is the gold standard, and WHO recommends that at least $70 \%$ of reported cases be confirmed using it in any survey [16]. After the 1998 International Conference on Buruli ulcer Control and Research in Yamoussoukro, Cote d' Ivoire [17], many endemic countries kicked-off their national control programmes. Unlike other nations, the BUD control programme in Nigeria is in its infancy and is yet to cover all endemic areas [17]; hence there is a gross information dearth about BUD among the populace and some healthcare practitioners. The programme suffers inadequate health infrastructure, low surveillance, under-reporting, and poor measures geared towards prevention and control [18]. This situation led to Nigerians seeking medical assistance in neighbouring countries [19]. The lack of health infrastructure in the country includes the nonexistence of a standard laboratory for PCR diagnosis of BUD. Hence, this study was embarked upon to assist the national control programme to overcome this challenge.

Most of the cases reported in the country had limitations, including using a purely descriptive approach, with most diagnoses being retrospective or prospective based only on clinical presentation $[20,21]$. This led to the speculation that BUD may be underdiagnosed hence under-reported in Nigeria compared to its two endemic neighbours on the east (Cameroon) and west (Benin republic). Therefore there is an urgent need to establish a PCR diagnosis laboratory to address this speculation, hence the need for this study.

\subsection{METHODOLOGY}

\subsection{Study Population}

From January 2016 to June 2018, samples were received from fifteen states from Southern Nigeria, with a few 
coming from the Federal Capital Territory (FCT), the country's central zone. The states were; Abia, Akwa Ibom, Anambra, Bayelsa, Cross Rivers, Delta, Ebonyi, Ekiti, Enugu, Imo, Lagos, Ogun, Ondo, Osun, and Rivers. A total of 920 samples from BUD suspected cases were screened during the study period.

\subsection{Study design}

The study was cross-sectional and community-based. Community Health Workers (CHWs) from the states were trained on clinical sample collection, storage and transportation to the laboratory. Before sample collection, there was outreach and sensitization of the communities, including distribution of information, education and communication (IEC) materials, interactive talks, and occasionally video shows. Patients were interviewed, and their details recorded before samples were collected. Trained CHWs took a swab and Fine Needle Aspiration (FNA) specimens. Collected samples were stored in appropriate transport media and sent to the laboratory for analysis.

\subsection{Ethics Statement}

Approval for the study was obtained from the Nigerian Institute of Medical Research, Institutional Review Board (IRB) (NO: IRB/15/314). The project was done in collaboration with the national BU control programme. Approval and participants' consent was also obtained at the state level where samples were received.

\subsection{Sample preparation and DNA isolation}

Specimens received on swab sticks were cut to size to fit into $2 \mathrm{ml}$ tubes, then $500 \mathrm{ml}$ of phosphate-buffered saline (PBS) was added and mixed vigorously for 5 minutes. One hundred microlitres of the solution was aliquoted into another sterile screw cap tube ready for DNA extraction. Fine Needle Aspirates (FNA), if dry on arrival, $200 \mu 1$ of PBS was added and mixed for 5 minutes, and $100 \mu 1$ aliquoted to sterile tubes. If FNA was in alcohol on arrival, it was centrifuged, the supernatant decanted, and $200 \mu 1$ of PBS added and mixed briefly, and $100 \mu 1$ aliquoted to a sterile tube, ready for DNA extraction.

For DNA extraction, Ethanol-Sodium Hydroxide (EtNa) $\{200 \mathrm{mM} \mathrm{NaOH}, 61 \%$ ethanol and $2.25 \mathrm{mM}$ EDTA $\}$ extraction protocol was used. Briefly, to $100 \mu 1$ of the bacterial suspension in PBS, 455ml of EtNa DNA extraction reagent was added and mixed briefly. The mixture was then heated at $95 \mathrm{oC}$ for 10 minutes, spun at $16,000 \mathrm{rpm}$ (using MIKRO 200, Hettich Zentrfugen centrifuge) for 10 minutes, and the supernatant was removed. The pellet was re-suspended in $100 \mu 1$ of DNA suspension solution. One-tenth volume of Sodium Acetate (3M, $\mathrm{pH}$ 5.2) was added and mixed, then $2 \mathrm{X}$ the volume of ice cold $100 \%$ ethanol was added and vortex for 10 seconds. It was then incubated overnight at $-20 \mathrm{oC}$. After the incubation, it was centrifuged at 13,000 rpm for 30 minutes, the supernatant discarded, rinsed by adding $250 \mu 1$ of ice-cold $70 \%$ ethanol, and centrifuged at 13,000 rpm for 10 minutes. The supernatant was discarded, and the pellet dissolved in 50ul of TE and used for PCR.

\subsection{DNA amplification}

$10 \mu 1$ of the extracted DNA was amplified in a $20 \mu 1$ reaction mixture containing $25 \mathrm{pmol}$ of each primer (pGp1 and pGp2), 1X FIREPol ${ }^{\circledR}$ Master mix ready to load, FIREPol ${ }^{\circledR}$ DNA Polymerase and $5 \mathrm{X}$ reaction buffer (0.4M Tris-HCl, 0.1M (NH4)2SO4, 0.1\% w/v Tween-20, 7.5mM MgCl2, 1mM dNTPs, Blue dye: $3.5-4.5 \mathrm{~kb}$ DNA fragment, Yellow dye: 35-45bp DNA fragment, a compound that increases sample density for direct loading). Primers used directed at IS2404 were pGp1 (5'AGGGCAGCGCGGTGATACGG 3') and pGp2 (5'CAGTGGATTGGTGCCGATCGAG 3'), with a thermocycling profile thus: denaturation at $95^{\circ} \mathrm{C}$ for 15 minutes; 40 cycles of $94^{\circ} \mathrm{C}$ for 30 seconds, $64^{\circ} \mathrm{C}$ for 1 minute, and $72^{\circ} \mathrm{C}$ for 1 minute 30 seconds and final elongation at $72^{\circ} \mathrm{C}$ for 10 minutes. The heated lid function was used at $105^{\circ} \mathrm{C}$ throughout the cycling time. Before the following step of the nested PCR, the status of the negative and positive controls was confirmed through agarose gel electrophoresis as indicated by the absence/ presence of 549-bp amplicon, which was a fragment indicating the presence of M. ulcerans genomic DNA. For the nested PCR, $3 \mu 1$ of the first amplicon was used in a $20 \mu \mathrm{l}$ reaction mixture with primers pGp3 (5'GGCGCAGATCAACTTCGCGGT-3') and pGp4 (5'CTGCGTGGTGCTTTACGCGC-3'). Other reaction components were added at similar concentrations with the first PCR. The thermocycling profile was also identical with the first except that the number of cycles was changed to 35 .

\subsection{Gel electrophoresis}

Two percent agarose gel in $0.5 \mathrm{X}$ TBE buffer was prepared with ethidium bromide. Ten microliters of each 
PCR product were loaded into the wells of the gel. On a row on the gel were a molecular weight standard (marker), positive and negative controls and test sample amplicons. The gels were run at $100 \mathrm{~V}$ for $1 \mathrm{hr}$ and were viewed in a photo documentation chamber (Clinx, USA) using UV light and captured on a laptop. Any 217-bp fragment appearing from the second PCR round was considered positive for M. ulcerans [22].

\subsection{Statistical analysis}

Categorical variables were summarized using frequencies. A Chi-squared test evaluated proportions of infection based on gender, age group, and states. Data analysis was done using SPSS 23.0

\section{RESULTS}

A total of 920 specimens were received during the study period (January 2016 - June 2018). The samples included those collected from microscopically positive and negative patients and also from clinically suspected cases. The demographic characteristics are shown in Table 1. Patients' distribution based on sex showed that 458 (49.7\%) were females while $462(50.2 \%)$ were males, indicating almost a balanced sex distribution. The infection rates were significantly different $(\mathrm{p}<0.01)$ between gender, with females being more infected. Patients older than 15years were 748 (81.3\%), while 172 (18.7\%) were children $\leq 15$ years. Infection rates were also significantly different between the age groups $(\mathrm{p}<0.05)$, with age groups $>15 y$ rs having more infection.

Table 1: Demographic characteristics of patients infected with M. ulcerans

\begin{tabular}{lllll}
\hline Variable & \multicolumn{4}{l}{ Infection rate } \\
& $\mathrm{n}$ & $(\%)$ & $\begin{array}{l}\text { Chi- } \\
\text { squared }\end{array}$ & p-value \\
Gender & & & & \\
Female $(\mathrm{n}=458)$ & 223 & 48.7 & 181.7 & $0.000^{*}$ \\
Male $(\mathrm{n}=462)$ & 204 & 44.2 & & \\
Age group & & & & \\
$<15$ years $(\mathrm{n}=172)$ & 99 & 57.6 & & \\
$16-25$ years $(\mathrm{n}=186)$ & 80 & 43.0 & & \\
$26-35$ years $(\mathrm{n}=113)$ & 54 & 48.2 & 17.94 & $0.022^{*}$ \\
$36-45$ years $(\mathrm{n}=151)$ & 59 & 39.1 & & \\
$>45$ years $(\mathrm{n}=298)$ & 135 & 45.3 & & \\
Total & 427 & 100.0 & & \\
& & & & \\
\hline
\end{tabular}

$* \mathrm{p}<0.05$ is considered significant
During the study period, the highest number of samples; $171(18.6 \%)$ and $170(18.5 \%)$ were received from Cross Rivers and Delta states, respectively. While the least number of samples; $2(0.2 \%)$ and $3(0.3 \%)$ came from Ekiti and Lagos states respectively, as shown in Figure 2

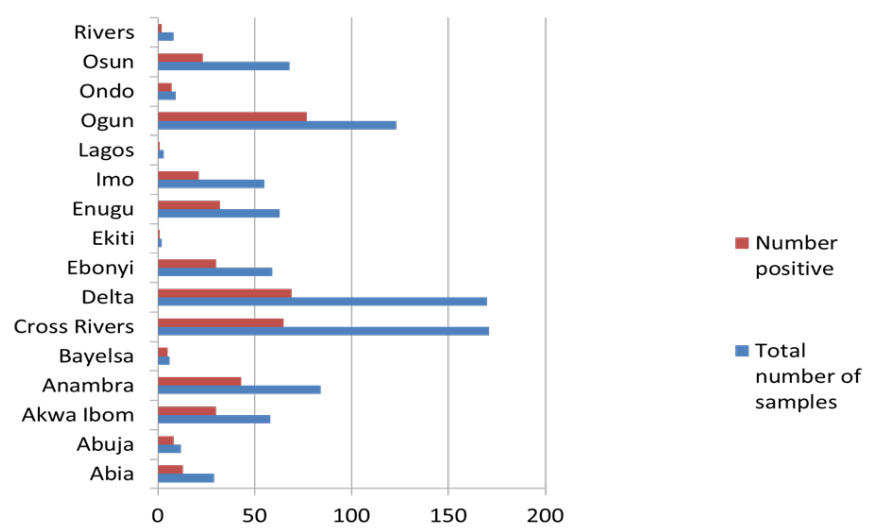

Figure 1. Distribution of M. ulcerans by states.

A total of 427 (46.4\%) out of 920 samples received were positive for M. ulcerans by PCR. Out of which, 204 were males, and 223 were females. An example of a gel showing both positive and negative samples is shown in Figure 2 . In the age group $\leq 15$ years, a total of 99 (57.6\%) out of 172 specimens were positive. While in ages above 15 years, out of 747 samples, 329 (44\%) were positive. Age group $>45$ years had the highest number (298; $32.4 \%$ ), of samples, of which $136(45.6 \%)$ were positive (Table 1).

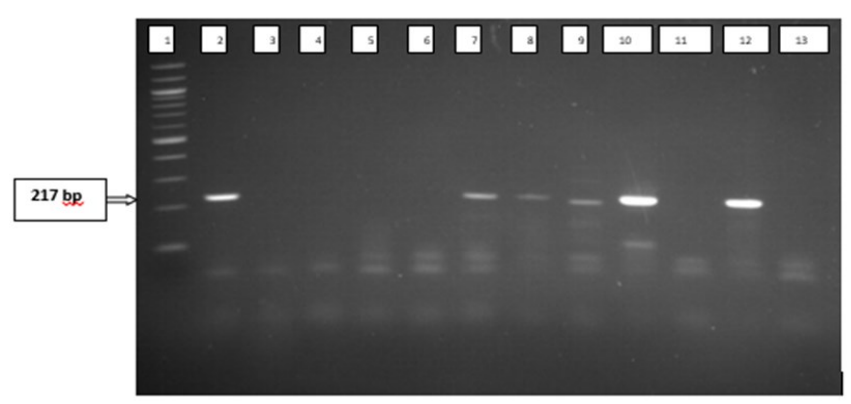

Figure 2. PCR for Mycobacterium ulcerans in clinical samples. Gel picture showing positive and negative results after second PCR. Lane $1=100 \mathrm{bp}$ marker, lane $2=$ positive sample, lanes 3-6 = negative samples, lanes 7-10 = positive samples, lane $11=$ negative sample, lane $12=$ positive control, lane 13 = negative control.

\section{Discussion}

Buruli Ulcer Disease (BUD) is a global health issue due to the pain, deformation, and gross economic burden on patients' families. In our study, out of the 920 specimens 
received, almost half, 427(46\%), were positive for $M$. ulcerans. Out of this number, 204 were males while 223 were females. This $46 \%$ prevalence recorded agrees with previous studies, which also showed that Southern Nigeria was BUD endemic $[14,19]$. Females were more infected than males $(48.7 \%$ and $44.2 \%$, respectively), which is in disagreement with reports elsewhere in Africa [23] and Japan [24]. Such gender variation has been attributed to differential exposure to M. ulcerans and immune responses $[23,25]$. Many studies have suggested that age group $<15$ years are mostly affected by BUD in sub-Saharan Africa $[19,23,26,27]$, but this is not in line with our study. Only 19\% (172) were under 15 years, which is the same as what was recorded in a study in Japan [24]. Over $80 \%$ of our study patients were aged $\geq 15$ years, with most samples coming from the age group $\geq 45$ years (32.4\%). A study by Ayelo et al, [28], also showed a similar outcome, but it cannot be generalized that most age group $\geq 15$ years are affected by BUD in Nigeria because other studies had contrary reports [19].

All samples examined were from the southern part of the country, except Abuja (in the central region). The southern region has similar climatic conditions with other BU endemic areas of sub-Saharan Africa, a tropical rainforest type. Other studies have also shown that this region is endemic $[29,30]$. Northern Nigeria is characterized by a tropical dry climate which has not been reported to be associated with BUD endemicity. The highest numbers of samples were from Cross Rivers (171) and Delta (170) states. Going by their names literally, these are areas with lots of water bodies and marshy environments known to support BU presence. Ekiti (2) and Lagos (3) had the lowest number of samples attributed to a lack of proper awareness of BU by the populace.

This study reports the first in-country nested PCR confirmed BU cases of a large cohort of patients. Before now, the national control programme cooperated with the TB and Leprosy control program called "National Tuberculosis, Buruli ulcer, and Leprosy Control Programme (NTBLCP)", sending samples of suspected cases to a WHO laboratory in Antwarp, Belgium, for PCR confirmation. This consumed a lot of time, leading to treatment delays, and required a lot of logistics. The IS2404 PCR for BU used in this project is considered the gold standard diagnostic tool [31]. It has high specificity and sensitivity with low detection limits [32-34].

\subsection{Conclusion}

This study has shown that southern Nigeria is endemic for BU, which agrees with other previous studies [14, 19] and supports the notion that BU's prevalence in Nigeria might be more than previously thought. The outcome of this study stresses the need for more government involvement in providing the needed health facilities to help alleviate the agony of BU patients.

\section{Declaration of conflict of interest}

The Authors declare that there is no conflict of interest.

\section{Funding Statement}

This study was supported by WHO/TDR impact grant (Project ID No. 235553), Nigerian Institute of Medical Research (grant number: 1nimrga0002-17-01), and an equipment grant from Kindermissions werk Aachen (Germany). A lot of Support also came from the German Leprosy and TB Relief Association (GLRA).

\section{Acknowledgment}

We thank all the community health workers (CHEWs) in the different study states, and Mrs. Adelaja and Miss Akpan of NIMR for their immense support during the study.

\section{Authors Contribution}

VPG contributed to study design, performed data collection, analysis and wrote the manuscript. TW performed data collection and analysis. SIA contributed to study design and data analysis. AO performed data collection and analysis. OPA conceived, designed and supervised the work. All authors approved the final version of the manuscript

\section{References}

1. WHO Buruli ulcer disease factsheet. Geneva: World Health Organization; 2016. http://www.who.int/ mediacentre/factsheets/fs199/en/[accessed 23 August 2016].

2. Stragier P, Ablordey A, Bayonne LM, Lugor YL, Sindani LS, Suykerbuyk P, et al. Heterogeneity among Mycobacterium ulcerans isolates from Africa. Emerg Infect Dis. 2006; 12(5): 844-847.

3. Clancey J, Dodge R, Lunn HF. Study of a mycobacterium causing skin ulceration in Uganda. Ann Soc Belg Med 
Trop. 1962; 4: 585-90.

4. Merritt RW, Walker ED, Small PL, Wallace JR, Johnson $\mathrm{PD}$, Benbow ME, et al. Ecology and transmission of Buruli ulcer disease : a systematic review. PloSNegl Trop Dis. 2010; 4(12): e911.

5. Einarsdottir T, Huygen K. Buruli ulcer. Human vaccines . 2011; 7(11): 1198-1203

6. Sakyi SA, Aboagye SY, Otchere ID, Liao AM, Caltagirone TG, Yeboah-Manu D. RNA Aptamer that specifically binds to mycolactone and serves as a diagnostic tool for diagnosis of Buruli ulcer. PloSNegl Trop Dis. 2016; 10 (10): e0004950.

7. Narh CA, Mosi L, Quaye C, Tay SC, Bonfoh B, de Souza DK. Genotyping tools for Mycobacterium ulcerans- Drawbacks and future prospects. Mycobact Dis. 2014; 4(2): 1000149

8. Komolafe OO. Buruli ulcer in Malawi-a first report. Malawi Medical Journal. 2001; 13(3): 37-39.

9. Huang GKL, Johnson PD. Epidemiology and management of Buruli ulcer. Expert Rev Anti Infect Ther. 2014; 12:855 -65 .

10. Hotez PJ, Kamath A. Neglected tropical diseases in SubSaharan Africa: review of their prevalence, distribution, and disease burden. PLoSNegl Trop Dis. 2009; 3:e412.

11. Zogo B, Djenontin A, Carolan K, Babonneau J, Guegan J$\mathrm{F}$, Eyangoh S, et al. A field study in Benin to investigate the role of mosquitoes and other flying insects in the ecology of Mycobacterium ulcerans. PLoS Negl Trop Dis. 2015; 9(7):e3941.

12. Eddyani M, Ofori-adjei D, Teugels G, De Weirdt D, Boakye D, Meyers WM, et al. Potential role of fish in transmission of Mycobacterium ulcerans disease (Buruli ulcer): an environmental study. Appl Environ Microbiol. 2004; 70(9): 5679-81.

13. Wilson MD, Boakye DA, Mosi L, Asiedu K. In the case of transmission of Mycobacterium ulcerans in buruli ulcer disease Acanthamoeba species stand accused. Ghana Med J [Internet]. 2011; 45(1): 31-4.

14. Chukwuekezie O, Ampadu E, Sopoh G, Dossou A, Tiendrebeogo A, Sadiq L, et al. Buruli ulcer, Nigeria. Emerg Infect Dis. 2007; 13(5): 782-4.

15. Oluwasanmi JO, Solankee TF, Olurin EO, Itayemi SO, Alabi GO, et al. Mycobacterium ulcerans (Buruli) skin ulceration in Nigeria. Am J Trop Med Hyg. 1976; 25:122128.

16. World Health Organization. Incidence, prevalence and mapping of Buruli ulcer. http://www.who.int/buruli/ research/priorities/healthmapping/en/(accessed September 9, 2015).
17. Organisation Mondiale de la Sante'. Conference internationale sur la lutte et la recherché relatives a' l' ulcere de Buruli, 6-8 juilet 1998. Yamoussoukro, Co'te d'Ivoire.

18. Oke AA, Komolafe IO, Akinwale O, Gyang P, Henry E, Nwafor T. Index cases of Buruli ulcer disease in three states of south west, Nigeria- a preliminary study. Journal of Pharmacy and Biological Sciences. 2018; 13(2): 51-56.

19. Marion ECK, Adeye A, Kempf M, Chauty A, Marsollier L. Buruli ulcer in South Western Nigeria: A retrospective cohort study of patients treated in Benin. PloSNegl Trop Dis. 2015; 9(1):e3443.

20. Oluwasanmi JO, Solankee TF, Olurin EO et al. Mycobacterium ulcerans (Buruli), skin ulceration in Nigeria. Am J Trop Med Hyg. 1976; 25:122-8.

21. Chukwuekezie O, Ampadu E, Sopoh G et al. Buruli ulcer, Nigeria. Emerg Infect Dis. 2007; 13:782-3.

22. Stienstra Y, van der Werf TS, Guarner J, Ragahunathan PL, Spotts Whitney EA, van der Graaf WTA, Asamoa K, Tappero JW, Ashford DA, King CH. Analysis of an IS2404-based PCR for diagnosis of Buruli Ulcer Disease in regions of Ghana where the disease is endemic. Journal of Clinical Microbiology. 2003; 41:794-797.

23. Vincent QB, Ardant MF, Adeye A et al. Clinical epidemiology of laboratory confirmed Buruli ulcer in Benin: a cohort study. Lancet Glob Health. 2014; 2:e422-430.

24. Nakanaga K, Hoshino Y, Yotsu RR, Makino M, Ishii N. Nineteen cases of Buruli ulcer diagnosed in Japan from 1980 to 2010. Journal of Clinical Microbiology. 2011; 49:3829-3836.

25. Pouillot R, Matias G, Wondje CM, Portaels F, Valin N et al. Risk factors for buruli ulcer: a case control study in Cameroon. PloS Negl Trop Dis. 2007; 1:e101.

26. Van der Werf TS, Stienstra Y, Johnson RC, Philips R, Adjei O, Fleischer B et al. Mycobacterium ulcerans disease. Bull World Health Organ. 2005; 83(10):785-91.

27. World Health Organization. Number of reported cases: Data by country. Geneva: The Organization ; 2017 [cited $2018 \mathrm{Feb} 8]$. http://apps.who.int/gho/data/ view.main.95000.

28. Ayelo GA, Anagonou E, Wadagni AC, Barogui YT, Dossou AD et al. Report of a series of 82 cases of Buruli ulcer from Nigeria treated in Benin, from 2006 to 2016. PloS Negl Trop Dis. 2018; 12(3): e0006358.

29. Vandelannoote K, Jordaens K, Bomans P, Leirs H, Durnez $\mathrm{L}$ et al. Insertion sequence element single nucleotide polymorphism typing provides insights into the population structure and evolution of Mycobacterium ulcerans across Africa. Appl Environ Microbiol. 2014; 80: 1197-1209.

30. Meka AO, Chukwu JN, Nwarfor CC, Oshi DC, Madichie 
NO, Ekeke N, Anyim MC et al. Diagnosis delay and duration of hospitalization of patients with Buruli ulcer in Nigeria. Trans R Soc Trop Med Hyg. 2016; 110: 502- 509.

31. World Health Organization, WHO Report Weekly, Epidemiology Record, May 14, 2004.

32. Guimaraes-Peres A, Portaels F, de Rijk P, Fissette K et al. Comparison of two PCRs for detection of Mycobacterium ulcerans. J. Clin. Microbiol. 1999; 37:206-208.
33. Ross BC, Johnson PD, Oppedisano F, Marino L, Sievers A, Stinear T et al. Detection of Mycobacterium ulcerans in environmental samples during an outbreak of ulcerative disease. Appl. Environ. Microbiol. 1997; 63: 4135-4138.

34. Stinear T, Davies JK, Jenkin GA, Hayman JA, Oppedisano F, Johnson PD. Identification of Mycobacterium ulcerans in the environment from regions in southeast Australia in which it is endemic with sequence capture-PCR. Appl. 